\title{
Febuxostat-associated rhabdomyolysis in chronic renal failure
}

\section{It is likely that \\ [his] statin \\ and fibrate \\ hypolipidaemic}

medications ...

contributed to

his myositis

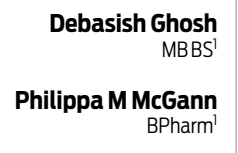

Timothy J Furlong PhD, FRACP

Richard O Day MD, FRACP ${ }^{1,2}$

1St Vincent's Hospital, Sydney, NSW.

2 St Vincent's Clinical School, UNSW, Sydney, NSW.

dbasis78@gmail.com

doi: 10.5694/mjal4.01404

\section{Clinical record}

A 68-year-old man of European descent presented to our emergency department with rhabdomyolysis and acute-onchronic kidney disease. He had a history of stage 3 chronic kidney disease (CKD3) - based on 17 tests in the year before this admission, his estimated glomerular filtration rate (eGFR) was $35 \pm 7 \mathrm{~mL} / \mathrm{min} / 1.73 \mathrm{~m}^{2}$ (CKD3: eGFR $=30-59 \mathrm{~mL} / \mathrm{min} / 1.73 \mathrm{~m}^{2}$ ), his serum creatinine concentration was $179 \pm 42 \mu \mathrm{mol} / \mathrm{L}$ (reference interval [RI], 60-120 $\mathrm{mol} / \mathrm{L}$ ) - and of polyarticular tophaceous gout, type 1 diabetes mellitus, hypertension, ischaemic heart disease (coronary artery bypass grafting in 1998) and peripheral vascular disease.

His gout, diagnosed 13 years before this presentation, had been treated with allopurinol and then colchicine. Both, however, caused anaphylactic reactions. He had had multiple short courses of prednisolone to treat acute attacks on a background of naproxen. A month before this presentation, naproxen was withdrawn and treatment with a new hypouricaemic drug, febuxostat (40 mg daily) initiated. This drug was obtained through the Special Access Scheme of the Therapeutic Goods Administration. Two doses of febuxostat were withheld 12 days before this admission when he was admitted to hospital for 8 days with Haemophilus influenzae pneumonia and acute-on-chronic kidney disease, with his serum creatinine concentration peaking at $245 \mu \mathrm{mol} / \mathrm{L}$. He was treated with intravenous ceftriaxone and azithromycin for 5 days, after which his renal function values had returned to baseline levels. He was discharged home and prescribed oral amoxicillin, which was to be continued for 5 days.

When he presented to our emergency department, the man was lethargic, oliguric, dehydrated and with acute-on-chronic kidney disease (serum creatinine concentration, $669 \mu \mathrm{mol} / \mathrm{L}$; eGFR, $7 \mathrm{~mL} / \mathrm{min} / 1.73 \mathrm{~m}^{2}$ ), but he was haemodynamically stable. There were no symptoms suggesting infection, and he was afebrile. Elevated serum creatine kinase activity (48200 U/L; RI, 20-200 U/L) and the presence of myoglobin in his urine were consistent with rhabdomyolysis. The man was hyperkalaemic (potassium, 6 mmol/L; RI, 3.5-5.0 mmol/L) with tall T waves in his electrocardiogram; this responded to oral resonium and nebulised salbutamol. His serum creatinine concentration continued to rise despite intravenous hydration, peaking 48 hours after admission at $833 \mathrm{\mu mol} / \mathrm{L}$ (eGFR, $6 \mathrm{~mL} / \mathrm{min} / 1.73 \mathrm{~m}^{2}$ ). His bicarbonate levels declined from $22 \mathrm{mmol} / \mathrm{L}$ to $15 \mathrm{mmol} / \mathrm{L}$ (RI, $24-31 \mathrm{mmol} / \mathrm{L})$, reflecting worsening metabolic acidosis.
At the time of his admission, he was taking (in addition to febuxostat) aspirin (100 mg daily), simvastatin (40 mg daily), gemfibrozil (600 mg twice daily), frusemide (40 mg daily), metoprolol (100 mg twice daily), moxonidine (200 mg twice daily), insulin and omeprazole (20 mg daily).

Febuxostat was considered to be the likely dominant precipitating factor and was withdrawn, as were simvastatin and gemfibrozil; he had used these two medications for 12 years, but febuxostat had only recently been prescribed. Further, application of the Naranjo adverse drug reaction (ADR) probability scale indicated that febuxostat possibly caused rhabdomyolysis in our patient.

Haemodialysis of the patient was commenced, and he had five cycles over the next 11 days. His renal function gradually returned to baseline (Figure). He was discharged on Day 23. Some months later, treatment with benzbromarone was started, a uricosuric drug that was also obtained through the Special Access Scheme. After taking benzbromarone for one-and-a-half months, his plasma urate concentration was $0.26 \mathrm{mmol} / \mathrm{L}$ ( $R \mathrm{l}$, 0.25-0.50 mmol/L).

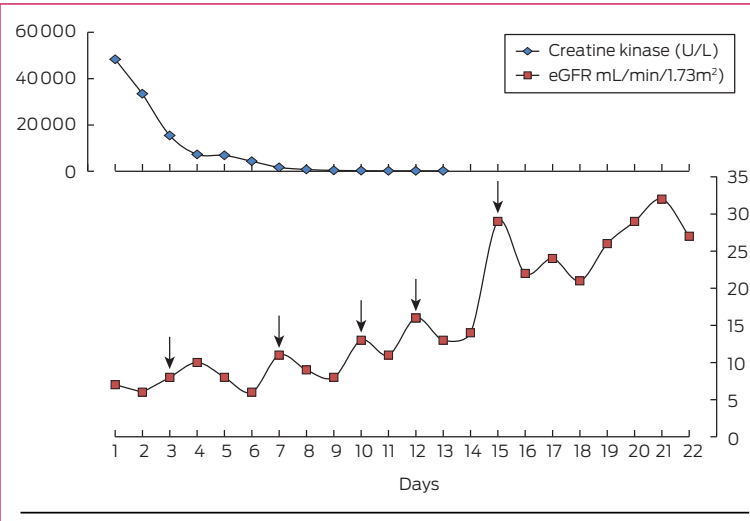

Time course of serum creatine kinase activity and estimated glomerular filtration rate (eGFR) in our patient. Arrows: Dialysis was undertaken on Days 3, 7, 10, 12 and 15 .
U ncontrolled gout is a significant disorder because of the debilitating attacks of acute gout, the ensuing joint damage that causes pain, deformity and loss of function, and the organ damage involved, particularly renal dysfunction. The xanthine oxidase inhibitor allopurinol is an effective medication for reducing plasma urate concentrations to below $0.36 \mathrm{mmol} / \mathrm{L}$, and this effect, if maintained, will almost always eliminate recurrent acute attacks of gout and the risk of joint and organ damage..$^{2-4}$ As the active form of the drug is cleared exclusively by the kidney, the starting dose needed by patients with impaired renal function is lower. ${ }^{4}$ Risk of hypersensitivity, manifested as toxic epidermal necrolysis, is an uncommon but significant problem; a macular-papular rash is seen in as many as $2 \%$ of patients. These problems may be more common in those with impaired renal function or also using diuretic medications. ${ }^{5}$

There are alternative approaches for lowering urate levels in patients with hypersensitivity reactions. Uricosuric drugs, such as probenecid, are effective in patients with normal to moderately impaired renal function, but are not effective in those with severe renal impairment. ${ }^{6}$ Benzbromarone is a more effective uricosuric agent in individuals with renal impairment, but must be imported into Australia under the Special Access Scheme, as it is not registered in this country.

The availability of a second xanthine oxidase inhibitor, febuxostat, is therefore welcome, especially for patients who do not tolerate allopurinol well. Febuxostat is 
registered in the United States and Europe, and was recently also registered in Australia (December 2014). The medication is generally well tolerated and dose adjustment is not necessary in patients with mild to moderate renal dysfunction. Liver function abnormalities and cardiovascular thrombotic reactions have been identified by postmarketing studies, but their incidence is very low. Only a few case reports have described hypersensitivity reactions. Cost is an issue, as febuxostat is more expensive than allopurinol, and should therefore not be the agent of first choice.

Rhabdomyolysis is noted as a rare side effect in the product information for febuxostat (following post-marketing experience), but there has only been a single published case report. ${ }^{8}$ In our patient, a serious additional decline in renal function was marked by substantially elevated creatine kinase activity, suggesting that rhabdomyolysis had caused this decline. It is likely that the concomitant statin and fibrate hypolipidaemic medications (ie, simvastatin and gemfibrozil) that the patient had taken uneventfully for several years contributed to his myositis. Dehydration associated with the patient's recent pneumonia probably also contributed to renal deterioration.

It is notable that in our case and that of Kang and colleagues, ${ }^{8}$ patients with chronic kidney disease had been taking statins both before and together with febuxostat. The combination of chronic kidney disease and statin therapy may represent a risk for febuxostat-induced rhabdomyolysis and renal injury.

The options for reducing this patient's plasma urate levels - a critical goal, given his deteriorating renal function, progressive joint damage, and recurrent and severely painful acute gout - are quite limited. Febuxostat is now
Lessons from practice

- Options for reducing plasma urate levels to prevent recurrent acute and tophaceous gout are limited, especially in patients with impaired renal function.

- Febuxostat is an effective alternative xanthine oxidase inhibitor to allopurinol.

- Although the product information for febuxostat indicates that there is no need for dose adjustment in patients with moderate renal impairment, prescribers need to be cautious.

- Chronic kidney disease and concomitant statin therapy may represent a risk for febuxostat-induced rhabdomyolysis and subsequent renal injury.

clearly contraindicated. Probenecid has limited efficacy when the glomerular filtration rate falls below $30 \mathrm{~mL} /$ minute. Benzbromarone, which is more effective than probenecid in patients with impaired renal function, can be obtained in Australia under the Special Access Scheme, and is a reasonable option. However, the patient's hepatic function would need to be carefully monitored, as the medication was withdrawn from the market in Europe and North America after rare reports of serious hepatic toxicity. ${ }^{7}$ Recombinant forms of uricase (such as rasburicase, pegloticase) would undoubtedly be effective in reducing urate levels, but repeated use would be prohibitively expensive; further, there is a risk of developing antibodies to pegloticase, which results in reduced efficacy and the possibility of adverse effects. ${ }^{9}$ Desensitisation to allopurinol, although complex, time-consuming and not without risk, also remains an option. ${ }^{10}$

Competing interests: No relevant disclosures.

References are available online at www.mja.com.au. 
1 Naranjo CA, Busto U, Sellers EM, et al. A method for estimating the probability of adverse drug reactions. Clin Pharmacol Ther 1981; 30: 239-245.

2 Jordan KM, Cameron JS, Snaith M, et al. British Society for Rheumatology and British Health Professionals in Rheumatology guideline for the management of gout. Rheumatology (Oxford) 2007; 46: 1372-1374.

3 Khanna D, Fitzgerald JD, Khanna PP, et al; American College of Rheumatology. 2012 American College of Rheumatology guidelines for management of gout. Part 1: systematic nonpharmacologic and pharmacologic therapeutic approaches to hyperuricemia. Arthritis Care Res (Hoboken) 2012; 64: 1431-1446.

4 Stamp LK, O’Donnell JL, Zhang M, et al. Using allopurinol above the dose based on creatinine clearance is effective and safe in patients with chronic gout, including those with renal impairment. Arthritis Rheum 2011; 63: 412-421.

5 Ramasamy SN, Korb-Wells CS, Kannagara DR, et al. Allopurinol hypersensitivity: a systematic review of all published cases, 1950-2012. Drug Saf 2013; 36: 953-980.
6 Day RO, Williams KM, Graham GG. Urate-lowering therapy: uricosurics. In: Dalbeth N, Perez-Ruiz F, Schlesinger N, editors. Gout. London: Future Medicine 2013: 174-188.

7 Lee MH, Graham GG, Williams KM, Day RO. A benefit-risk assessment of benzbromarone in the treatment of gout. Was its withdrawal from the market in the best interest of patients? Drug Saf 2008; 31: 643-665.

8 Kang Y, Kim MJ, Jang HN, et al. Rhabdomyolysis associated with initiation of febuxostat therapy for hyperuricaemia in a patient with chronic kidney disease. J Clin Pharm Ther 2014; 39 : 328-333.

9 Garay RP, El-Gewely MR, Labaune JP, Richette P. Therapeutic perspectives on uricases for gout. Joint Bone Spine 2012; 79: 237-242.

10 Colagiuri G, Nettis E, Di Leo E, et al. Allopurinol hypersensitivity reactions: desensitization strategies and new therapeutic alternative molecules. Inflamm Allergy Drug Targets 2013; 12: 19-28. 